\title{
Psychotic Symptoms of Hashimoto's Encephalopathy: A Diagnostic Challenge
}

\author{
Monisha K Savarimuthu, Sherab Tsheringla, and Priya Mammen \\ Department of Psychiatry, Christian Medical College, Mental Health Center, Vellore, India
}

Hashimoto's encephalopathy (HE) is a rare and underdiagnosed neuropsychiatric illness. We present the case of a 17-year-old girl who was admitted to a tertiary-care psychiatric center with acute onset psychosis and fever. Her psychotic symptoms were characterized by persecutory and referential delusions, as well as tactile and visual hallucinations. Her acute behavioral disturbance warranted admission and treatment in a psychiatric setting (risperidone tablets, $3 \mathrm{mg} /$ day). She had experienced an episode of fever with a unilateral visual acuity defect approximately 3 years before admission, which was resolved with treatment. Focused clinical examination revealed an enlarged thyroid, and baseline blood investigations, including thyroid function test results were normal. Abnormal laboratory investigations revealed elevated anti-thyroid peroxidase (anti-TPO) and anti-thyroglobulin (anti-TG) levels (anti-TPO of $480 \mathrm{IU} /$ $\mathrm{mL}$; anti-TG of $287 \mathrm{IU} / \mathrm{mL}$ ). Results of other investigations for infection, including cerebrospinal fluid examination, electroencephalography, and brain magnetic resonance imaging were normal. She was diagnosed with HE and was treated with intravenous corticosteroids (methylprednisolone up to $1 \mathrm{~g}$ /day; tapered and discontinued after a month). The patient achieved complete remission of psychotic symptoms and normalization of the anti-thyroid antibody titers. Currently, at the seventh month of follow-up, the patient is doing well. This case highlights the fact that in the absence of well-defined clinical diagnostic criteria, a high index of suspicion is required for early diagnosis of HE. Psychiatrists need to explore for organic etiologies when dealing with acute psychiatric symptoms in a younger age group.

Key Words: Hashimoto's encephalopathy; Steroid responsive encephalopathy associated with autoimmune thyroiditis; Risperidone; Steroid; Methylprednisolone; Psychosis; Anti-thyroid antibodies.

Received: August 7, 2018 / Revision: August 25, 2018 / Accepted: September 6, 2018

Address for correspondence: Sherab Tsheringla, Department of Psychiatry, Christian Medical College, Mental Health Center, Vellore, Tamil Nadu 632002, India

Tel: +91-4162282400, E-mail: sherabla@gmail.com

\section{INTRODUCTION}

Hashimoto's encephalopathy (HE) is a rare and underdiagnosed illness with worldwide prevalence rates of 2 to 3 per 100000 [1]. Globally, 130 cases have been reported [1]. Pediatric cases are under-reported, probably as a result of frequent underdiagnosis [2].

\section{Eponym}

Steroid responsive encephalopathy associated with autoimmune thyroiditis (SREAT) and encephalopathy associated with autoimmune thyroid disease (EAATD).

\section{CASE VIGNETTE}

A 17-year old girl was admitted to our tertiary care psychi-

This is an Open Access article distributed under the terms of the Creative Commons Attribution Non-Commercial License (https://creativecommons.org/licenses/by-nc/4.0) which permits unrestricted non-commercial use, distribution, and reproduction in any medium, provided the original work is properly cited. atric center with fever that lasted 1 week, a headache, bilateral blurred vision and psychosis (which included tactile hallucinations, visual hallucinations, persecutory and referential delusions). Three years prior to admission, she experienced a fever episode with a unilateral right visual acuity defect and right divergent squint, which was partially resolved with steroid treatment for three weeks. Physical examination findings revealed an enlarged thyroid, a short fourth toe on the left leg, a single transverse palmar crease in the right hand. No other neurological or eye findings were present. The fundus bore an old scar extending from the disc to the macula in her right eye with disc pallor. A mental status examination revealed visual hallucinations seeing monkeys and snakes running around. Delusions of persecution and reference were also present. PANS Scale (P-13; N-7; G-20; T-40). Informed consent has been provided by the family members.

The clinical differentials included inflammation, infectious, structural, traumatic, metabolic, genetic and neurodegenerative conditions including infectious and autoimmune 
Table 1. Hashimoto's encephalopathy work up in our patient

\begin{tabular}{ll}
\hline Baseline blood investigations & Intracranial infections (blood borne virus screen, measles, and PCR for viruses) \\
Thyroid function tests & Autoimmune \\
CSF, EEG, and MRI brain & Karyotyping \\
Inborn errors of metabolism & Wilsons work up, ceruloplasmin and slit lamp for KF rings \\
USG abdomen for germ cell tumours & Tumour markers-AFP, Beta hCG
\end{tabular}

AFP: alpha-fetoprotein, CSF: cerebrospinal fluid, EEG: electroencephalography, hCG: human chorionic gonadotropin, PCR: polymerase chain reaction, USG: ultrasound guidance

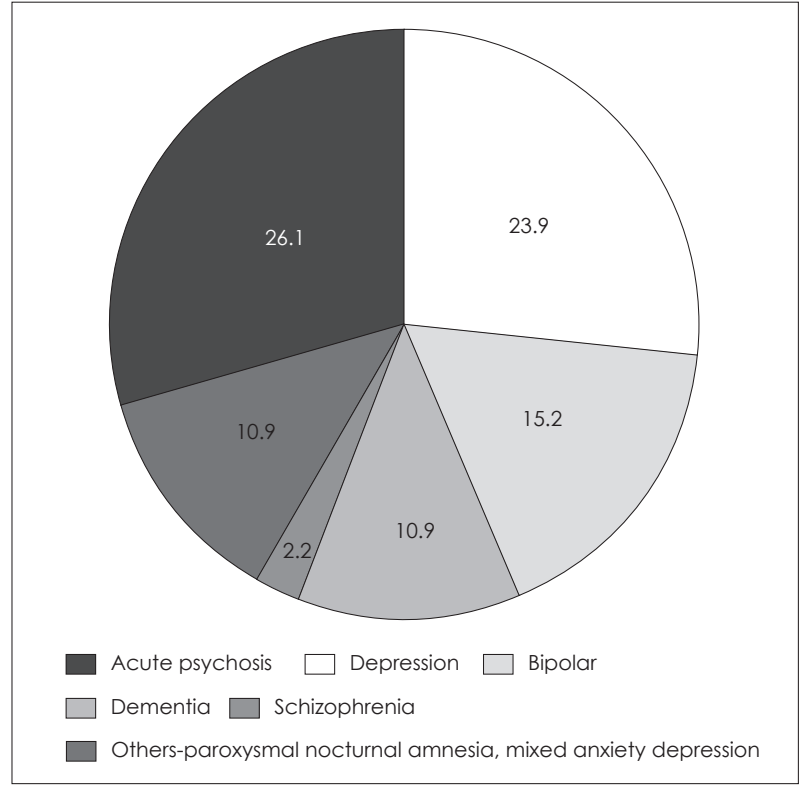

Fig. 1. Psychiatric manifestations in Hashimoto's encephalopathy [3].

enecephalitis.

Positive results included elevated anti-thyroid peroxidase $(480 \mathrm{IU} / \mathrm{mL})$ and anti-thyroglobulin $(287 \mathrm{IU} / \mathrm{mL})$ antibodies, a slit lamp examination revealed an old toxoplasma scar and visual evoked potentials of bilateral optic neuritis. The rest of the results in Table 1 were normal.

She was diagnosed with HE and treated with intravenous methylprednisolone (1 g/day; tapered and discontinued after 1 month) and symptomatically treated with antipsychotics. Diagnosis was made based on positive antithyroid peroxidase antibodies and neuropsychiatric features. Complete resolution of psychoses was observed within 1 month, with normalization of the anti-thyroid antibody titers. The patient is currently doing well, as established at the many months of follow up.

\section{DISCUSSION}

HE was first reported in 1966 by Lord Brain [4]. It derives its name from Hashimoto's thyroiditis, which is accompanied by anti-thyroid antibodies causing central nervous sys-

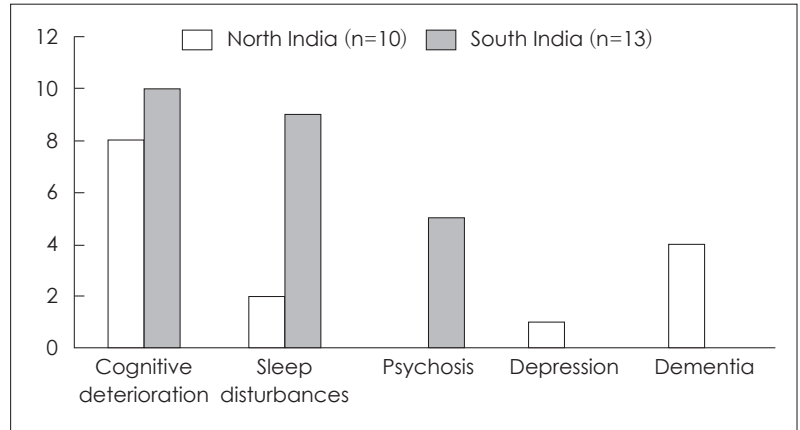

Fig. 2. Comparision of psychiatric features of Hashimoto's encephalopathy in 2 Indian studies [6].

Table 2. Neurological features of Hashimoto's encephalopathy [1]

\begin{tabular}{l}
\hline \multicolumn{1}{c}{ Neurological features } \\
\hline 1. Encephalopathy \\
Diffuse hyperreflexia (85\%) \\
Tremors \\
Myoclonus/seizures \\
2. Cerebellopathy-ataxia \\
3. Isolated myelopathy \\
\hline
\end{tabular}

Table 3. Neuropsychiatric presentations in Hashimoto's encephalopathy

\begin{tabular}{ll}
\hline \multicolumn{1}{c}{ Acute $(25 \%)$} & \multicolumn{1}{c}{ Chronic $(75 \%)$} \\
\hline Seizures $(>95 \%)$ & Cognitive deterioration \\
Stroke $(>65 \%)$ & Depression \\
Acute encephalopathy & Tremors \\
Psychosis (visual hallucinations $>90 \%)$ & Myoclonus \\
\hline
\end{tabular}

tem manifestations.

The proposed mechanisms include immune complex deposition (a complex of antigen and anti-thyroid antibody) in the brain vessel wall, causing vasculitis, which in turn, causes brain cell infarction $[1,5]$. Direct brain cell injury occurs because of the presence of antibodies against $\mathrm{NH}_{2}$ terminal alpha enolase (NAE), a common antigen in the brain and endothelial cells of the vessel wall [5].

HE is usually seen in the fifth decade of life, although a double peak has been described with the first peak around the second decade [1]. Female individuals have a higher propensity to develop HE with a sex ratio of 4:1 [1]. Onset of HE could be acute or subacute, with a progressive or relapsing-remit- 
Table 4. Investigations in Hashimoto's encephalopathy $[1,3,5]$ Varied, hypothyroidism commoner $[1,5]$

\begin{tabular}{|c|c|}
\hline \multicolumn{2}{|l|}{ TFT } \\
\hline $\begin{array}{l}\text { Thyroid peroxidase } \\
\text { antibodies }\end{array}$ & $\begin{array}{l}>1000 \text { IU/L, not a specific marker, } \\
\text { epiphenomenon of another } \\
\text { autoimmune process [4] }\end{array}$ \\
\hline Anti NAE antibodies & Specificity 91\% [5] \\
\hline CSF & Elevated proteins, lymphocytes [1] \\
\hline EEG & Diffuse slowing (most common) [1] \\
\hline MRI & Nonspecific [1] \\
\hline MRA & Narrowing of cerebral blood vessels [1] \\
\hline
\end{tabular}

CSF: cerebrospinal fluid, EEG: electroencephalography, NAE: $\mathrm{NH}_{2}$ terminal alpha enolase, MRA: MR angiography, TFT: thyroid function test

ting course [1]. Thyroid complications vary, with $55 \%$ of patients presenting with hypothyroidism $[1,3]$. Clinical features are non-specific and are highlighted in Table 2 and Figs. 1,2. Acute presentations are mostly neurological, whereas chronic presentations are mostly psychiatric (Table 3) [1]. Among the cases of acute psychosis, visual hallucinations have been reported with a frequency of approximately $90 \%$ [4].

Typical symptoms of HE in our patient included acute presentation of fever, visual hallucinations, acute encephalopathy, and recurrent stroke lasting for a short period of time. Typical manifestations included an enlarged thyroid gland, bilateral blurred vision, altered mental status, and visual hallucinations. An atypical observation included progression of psychosis to referential delusions.

$\mathrm{HE}$ is a diagnosis of exclusion as there is a lack of well-defined criteria for diagnosis or treatment. Organic etiologies need to be ruled out, as highlighted in the case section. Our clinical suspicion was high given the severity of symptoms (recurrent eye strokes with psychosis) and the presence of an enlarged thyroid gland (despite normal thyroid functions). The combination of thyroid disorder and neuropsychiatric symptoms led us to further investigate with anti-thyroid antibodies.

Other rare presentations of acute psychosis include conditions like neuropsychiatric manifestations of Japanese encephalitis, voltage gated potassium channel encephalitis, megaloencephalopathy leukoencephalopathy.

Table 4 highlights the complete panel of investigations that aided in diagnosis. Note that the most specific marker thus far is the anti-NAE antibody, with a specificity of around $91 \%$, and it is currently only used in research settings [4].

Treatment mainly includes the use of immunomodulators, of which glucocorticoids are the most commonly used [1]. Other uses of agents such as azathioprine and metho- trexate have been reported [1]. Furthermore, treatment with plasmapheresis has also been tested. Acute encephalopathies respond rapidly to immunomodulators [4]. In the event of acute strokes, appropriate treatment for strokes must be ensured. The use of corticosteroids for acute stroke remains controversial, probably because steroid treatment does not reverse stroke [1]. Long term treatment with steroids is proposed as a prophylactic treatment; however, the long-term side effects of steroids need to be considered when contemplating the use of prophylactic agents [1].

\section{CONCLUSION}

HE is diagnosed more often in autoimmune clinics and tertiary centers and less commonly in an outpatient psychiatric clinic during its initial presentation. We recommend clinicians to have a high index of suspicion for 1) young onset psychosis, follow up on clinical and laboratory thyroid tests and if abnormal explore autoimmune pathology. 2) severe presentation include neurological features pointing to enecephalitis. A simplistic diagnostic triad includes neuropsychiatric features, elevated anti thyroid antibodies, and response to steroids. Early identification is crucial for a favorable prognosis, as the disease is completely reversible with appropriate treatment.

\section{Conflicts of Interest}

The authors have no financial conflicts of interest.

\section{REFERENCES}

1) Sharma PM, Javali M, Mahale R, Madhusudhan BK, Majeed AA, Srinivasa R. Hashimoto encephalopathy: a study of the clinical profile, radiological and electrophysiological correlation in a Tertiary Care Center in South India. J Neurosci Rural Pract 2015;6:309-314.

2) Patnaik SK, Upreti V, D Dhull P. Steroid responsive encephalopathy associated with autoimmune thyroiditis (SREAT) in childhood. J Pediatr Endocrinol Metab 2014;27:737-744.

3) Menon V, Subramanian K, Tamizh JS. Psychiatric presentations heralding Hashimoto's encephalopathy: a systematic review and analysis of cases reported in literature. J Neurosci Rural Pract 2017; 8:261-267.

4) Sadock BJ, Sadock VA, Ruiz P. Kaplan and Sadock's comprehensive textbook of psychiatry. 10th ed. Philadelphia: Wolters Kluwer; 2017.

5) de Holanda NC, de Lima DD, Cavalcanti TB, Lucena CS, Bandeira F. Hashimoto's encephalopathy: systematic review of the literature and an additional case. J Neuropsychiatry Clin Neurosci 2011;23: 384-390.

6) Das M, Kayal AK, Goswami M, Basumatary LJ, Bhowmick S, Synmon B. Spectrum of Hashimoto's encephalopathy-An experience from the North East India. Indian J Med Spec 2016;7:71-75. 